Article

\title{
Brominated Bisindole Alkaloids from the Celtic Sea Sponge Spongosorites calcicola
}

\author{
Laurence K. Jennings ${ }^{1,2} \mathbb{D}_{\text {, Neyaz M. D. Khan }}^{3}$, Navdeep Kaur ${ }^{1,2}$, Daniel Rodrigues ${ }^{1,2} \mathbb{D}$, \\ Christine Morrow ${ }^{4}$, Aoife Boyd ${ }^{3}$ and Olivier P. Thomas $1, * \mathbb{C}$ \\ 1 Marine Biodiscovery Laboratory, School of Chemistry and Ryan Institute, National University of Ireland \\ Galway, University Road, Galway H91 TK33, Ireland; laurence.jennings@nuigalway.ie (L.K.J.); \\ navdeep.kaur@nuigalway.ie (N.K.); daniel.rodrigues@nuigalway.ie (D.R.) \\ 2 National Marine Biodiscovery Laboratory of Ireland, Marine Institute, Renville West, \\ Oranmore H91 R673, Ireland \\ 3 Discipline of Microbiology, School of Natural Sciences, Centre for One Health (Ryan Institute), Infection and \\ Immunity Cluster (NCBES), National University of Ireland Galway, University Road, Galway H91 TK33, \\ Ireland; m.khan5@nuigalway.ie (N.M.D.K.); aoife.boyd@nuigalway.ie (A.B.) \\ 4 Department of Natural Sciences, National Museums Northern Ireland, 153 Bangor Road, \\ Hollywood BT18 0EU, Ireland; christine.morrow@nmni.com \\ * Correspondence: olivier.thomas@nuigalway.ie; Tel.: +353-(0)-8-9455-6289
}

Academic Editors: Artur Silva and Diana Cláudia Pinto

Received: 15 October 2019; Accepted: 24 October 2019; Published: 29 October 2019

\begin{abstract}
As part of an ongoing program to identify new bioactive compounds from Irish marine bioresources, we selected the subtidal sponge Spongosorites calcicola for chemical study, as fractions of this species displayed interesting cytotoxic bioactivities and chemical profiles. The first chemical investigation of this marine species led to the discovery of two new bisindole alkaloids of the topsentin family, together with six other known indole alkaloids. Missing the usual central core featured by the representatives of these marine natural products, the new metabolites may represent key biosynthetic intermediates for other known bisindoles. These compounds were found to exhibit weak cytotoxic activity against HeLa tumour cells, suggesting a specificity towards previously screened carcinoma and leukaemia cells.
\end{abstract}

Keywords: marine natural products; sponge; cytotoxicity; bisindole alkaloid; Spongosorites

\section{Introduction}

Marine sponges are well-recognised as producers of a diverse array of bioactive metabolites for defence, competition, and communication [1]. For this reason, these invertebrates have been the subjects of extensive chemical study over the past half century. However, there still remain extensive regions of the world where sponges have been understudied, including much of the Irish coastal waters [2]. As part of an Irish marine biodiscovery initiative supported by the Marine Institute, invertebrates present in the shallow waters of Ireland have been inventoried and then chemically and biologically screened in the search for bioactive natural products. A priority list of species placed the sponge Spongosorites calcicola on the top of the list due to the amount of biomass available, good chemical profiles and interesting cytotoxic activity (Table S2). While this sponge species has never been studied chemically, other species of this genus have already led to interesting bioactive metabolites.

The sponge genus Spongosorites has been reported to produce many brominated bisindole alkaloids belonging to four classes that differ in the linkage between the indole moieties $[3,4]$. These bisindoles include topsentin and spongotine derivatives with imidazole or dihydroimidazole linkages [5,6], and also, dragmacidin and hamacanthin derivatives characterised by a pyrazine or piperazine 
central core [7-9]. These bisindole alkaloids have been largely studied due to their diverse array of bioactivities [10-12]. Herein, we describe the isolation, structure elucidation and cytotoxic activity of two new bisindole alkaloids from the Irish sponge $S$. calcicola featuring, for the first time, an absence of cycle between the two indole rings. As such, they could represent key intermediates in the biosynthesis of known bisindole derivatives.

\section{Results and Discussion}

The freeze-dried sponge material (5.2 g) was exhaustively extracted with a mixture of $\mathrm{MeOH} / \mathrm{CH}_{2} \mathrm{Cl}_{2}$ (1:1). The resulting extract (0.97 g) was fractionated using $\mathrm{C}_{18}$-SPE eluting with solvent mixtures from $\mathrm{H}_{2} \mathrm{O}$ to $\mathrm{MeOH}$ to DCM. The methanol fractions, containing several brominated alkaloids as evidenced in the (+)-HRESIMS spectra, were then subjected to successive reverse-phase HPLC purifications to yield the new calcicamides A and B (1-2) as TFA salts (Figure 1). These compounds were isolated with four other known brominated bisindole alkaloids isolated from species of the genus Spongosorites: trans-3,4-dihydrohamacanthin A (3) [13], 6-bromodeoxytopsentin (4), 6-bromotopsentin (5) $[13,14]$ and spongotine A (6) [5]. Additionally, two known monoindole derivatives related to the biosynthetic precursors were isolated from the same sample: $2-(1 H$-indol-3-yl)-2-oxoacetate methyl ester (7) and 2-(6-bromo-1H-indol-3-yl)-2-oxoacetate methyl ester (8).

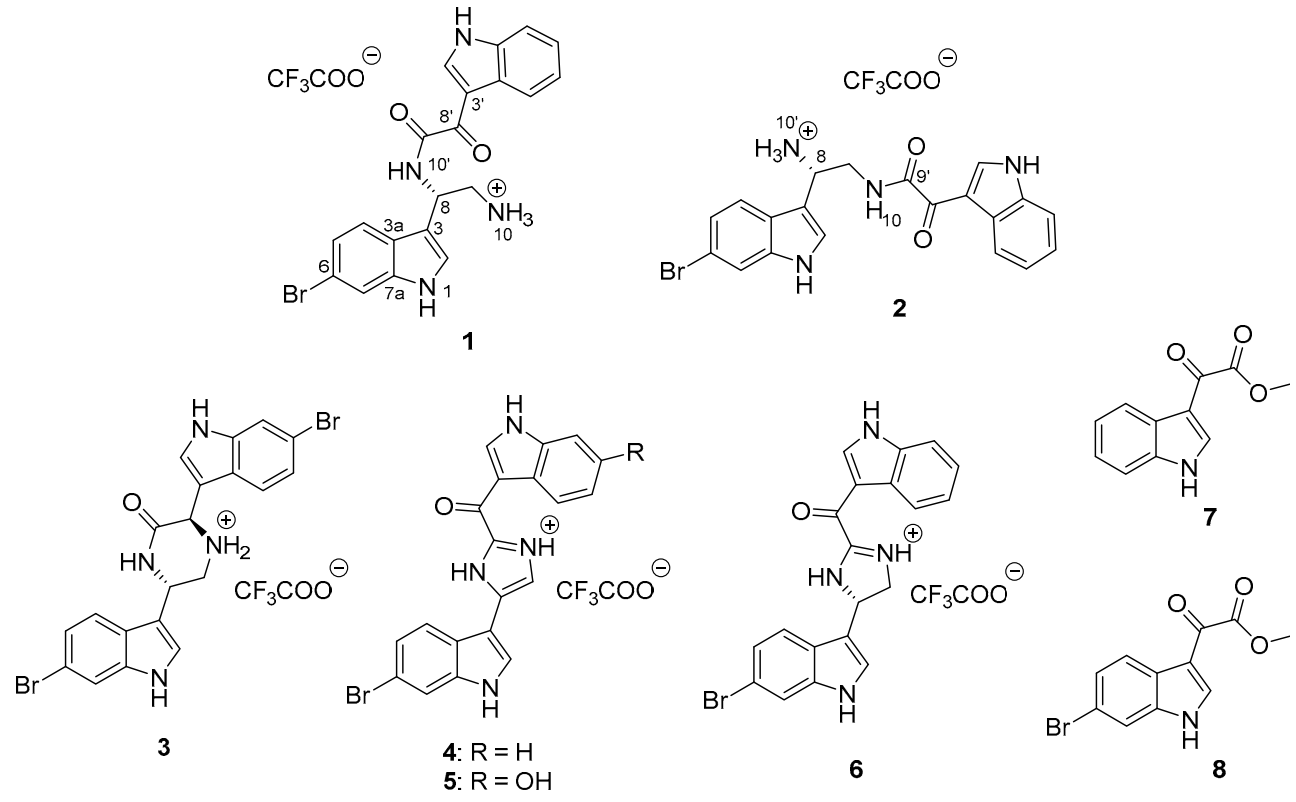

Figure 1. New calcicamides A and B (1-2) and known indole alkaloids, trans-3,4-dihydrohamacanthin A (3), 6-bromodeoxytopsentin (4), 6-bromotopsentin (5), spongotine A (6), 2-(1H-indol-3-yl)-2-oxoacetate methyl ester (7) and 2-(6-bromo-1H-indol-3-yl)-2-oxoacetate methyl ester (8) from Spongosorites calcicola.

Compound 1 was isolated as a bright yellow amorphous solid and displayed an ion cluster in the (+)-HRESIMS spectrum for $[\mathrm{M}+\mathrm{H}]^{+}$at $m / z$ 425.0614/427.0595 (1:1 ratio), consistent with the molecular formula $\mathrm{C}_{20} \mathrm{H}_{18} \mathrm{BrN}_{4} \mathrm{O}_{2}$. The ${ }^{1} \mathrm{H}-\mathrm{NMR}$ and ${ }^{13} \mathrm{C}-\mathrm{NMR}$ spectra performed in DMSO- $d_{6}$ displayed resonances associated with one indole at $\delta_{\mathrm{H}}$ 12.27, 8.80, 8.22, 7.53, 7.25, 7.23 and one 6-bromoindole at $\delta_{\mathrm{H}} 11.29,7.43,7.64,7.58,7.18$ (Table 1) [14]. The remaining signals of the ${ }^{1} \mathrm{H}-\mathrm{NMR}$ spectrum included one amide signal at $\delta_{\mathrm{H}} 9.15\left(\mathrm{~d}, J=9.2 \mathrm{~Hz}, \mathrm{NH}-10^{\prime}\right)$, one primary amine signal at $\delta_{\mathrm{H}} 7.98\left(\mathrm{br} \mathrm{s}, \mathrm{NH}_{3}-10\right)$, a nitrogenated methine at $\delta_{\mathrm{H}} 5.55(\mathrm{td}, J=9.3,4.6 \mathrm{~Hz}, \mathrm{H}-8)$ and a nitrogenated methylene at $\delta_{\mathrm{H}} 3.49$, $3.36\left(\mathrm{H}_{2}-9\right)$. A spin-coupled system between protons $\mathrm{H}-10^{\prime} / \mathrm{H}-8 / \mathrm{H}_{2}-9 / \mathrm{H}_{3}-10$ could be assigned from the COSY spectrum. HMBC correlations from $\mathrm{H}-8$ to C-2, C-3 and C-3a linked this spin coupled system to the bromoindole moiety. Finally, the ${ }^{13} \mathrm{C}-\mathrm{NMR}$ data indicated only two more resonances, associated with two carbonyls at $\delta_{C} 181.2$ and 163.1 (C-8' and C-9'), left to be assigned. The carbonyl 
chemical shifts and $\mathrm{HMBC}$ correlations from $\mathrm{H}-8$ and $\mathrm{H}-10^{\prime}$ to $\mathrm{C}-9^{\prime}$ were in agreement with an alpha keto amide substitution on the non-brominated indole. Even though no HMBC correlations to the C- $8^{\prime}$ carbonyl were observed, a ketone functional group at this position was supported by the presence of two absorption bands in the IR spectrum at 1672 and $1627 \mathrm{~cm}^{-1}$ [15]. With the 2D structure of 1 solved, the absolute configuration of the unique C-8 chiral centre was assigned through the comparison between experimental and predicted electronic circular dichroism (ECD) spectra (Figure 2). The ECD spectra of both enantiomers of $\mathbf{1}$ were calculated using Time-Dependent Density Functional Theory (TDDFT) at the B3LYP/6-311+G(2d,p)//B3LYP/6-311(2d,p) level of theory. As the experimental ECD spectrum had a close match with the calculated spectrum of the $8 S$ enantiomer of $\mathbf{1}$, we could assign the absolute configuration as $S$.

Compound 2 was isolated as a yellow amorphous solid that displayed an ion cluster in the (+)-HRESIMS for $[\mathrm{M}+\mathrm{H}]^{+}$at $m / z 425.0608 / 427.0590$, indicative of a structural isomer of $\mathbf{1}$. The ${ }^{1} \mathrm{H}-\mathrm{NMR}$ and ${ }^{13} \mathrm{C}-\mathrm{NMR}$ data (Table 1 ) were similar to those of $\mathbf{1}$, again revealing resonances associated with one indole $\left(\delta_{\mathrm{H}} 12.26,8.77,8.22,7.54,7.27,7.25\right)$ and one 6-bromoindole $\left(\delta_{\mathrm{H}} 11.45,7.43,7.71,7.63\right.$, 7.23). The differences between 1 and 2 were therefore located in the linkage between the two indole moieties. The ${ }^{13} \mathrm{C}-\mathrm{NMR}$ and IR data indicated the presence of an alpha-keto amide connected to the non-brominated indole. However, the spin-coupled system assigned through the COSY/HSQC spectra led us to identify the difference between 1 and 2 with the linkage from $\delta_{\mathrm{H}} 8.95(\mathrm{NH}-10)$ to $\delta_{\mathrm{H}} 3.83$, $3.70\left(\mathrm{H}_{2}-9\right)$ to $\delta_{\mathrm{H}} 4.83(\mathrm{H}-8)$ to $\delta_{\mathrm{H}} 8.33\left(\mathrm{NH}_{3}-10^{\prime}\right)$. The absolute configuration of the $\mathrm{C}-8$ stereocenter was again determined by ECD analysis. The theoretical ECD spectra of the enantiomers of 2 were calculated using the same methods and basis set/functional combination as used for $\mathbf{1}$, allowing the assignment of the same $8 S$ configuration for calcicamide B (Figure S15).

Table 1. NMR data for 1 and 2 in DMSO- $d_{6}\left({ }^{1} \mathrm{H}-\mathrm{NMR}\right.$ at $500 \mathrm{MHz}$ and ${ }^{13} \mathrm{C}-\mathrm{NMR}$ at $\left.125 \mathrm{MHz}\right)$.

\begin{tabular}{|c|c|c|c|c|c|c|}
\hline \multirow{2}{*}{ Pos. } & \multicolumn{3}{|c|}{1} & \multicolumn{3}{|c|}{2} \\
\hline & $\delta_{C}$, type & $\delta_{H}(J \cdot$ in $\mathrm{Hz})$ & НМВС & $\delta_{C}$, type & $\delta_{H}(J \cdot$ in $\cdot H z)$ & НMBC \\
\hline 1 & & $11.29, \mathrm{~d}(2.3)$ & $2,3,3 a, 7 a$ & & $11.45, \mathrm{~d}(2.2)$ & $2,3,3 a, 7 a$ \\
\hline 2 & 124.6, $\mathrm{CH}$ & $7.43, \mathrm{~d}(2.3)$ & $3,3 a, 7 a$ & 125.3, CH & $7.58, \mathrm{~d}(2.6)$ & $3,3 a, 7 a$ \\
\hline 3 & $112.6, \mathrm{C}$ & & & 109.6, C & & \\
\hline $3 a$ & $124.5, \mathrm{C}$ & & & 124.7, C & & \\
\hline 4 & $120.3, \mathrm{CH}$ & $7.64, \mathrm{~d}(8.7)$ & $3,6,7 a$ & $120.3, \mathrm{CH}$ & $7.71, \mathrm{~d}(8.5)$ & $3,6,7 a$ \\
\hline 5 & $121.8, \mathrm{CH}$ & $7.18, \mathrm{dd}(8.7,1.9)$ & $3 a, 6,7$ & $122.2, \mathrm{CH}$ & $7.23, \mathrm{dd}(8.5,1.7)$ & $3 a, 6,7$ \\
\hline 6 & $114.2, \mathrm{C}$ & & & $114.5, \mathrm{C}$ & & \\
\hline 7 & 114.3, $\mathrm{CH}$ & $7.58, \mathrm{~d}(1.9)$ & $3 a, 5,6$ & $114.6, \mathrm{CH}$ & $7.63, \mathrm{~d}(1.7)$ & $3 a, 5,6$ \\
\hline $7 a$ & 137.0, C & & & $136.8, \mathrm{C}$ & & \\
\hline 8 & $42.7, \mathrm{CH}$ & $5.55, \operatorname{td}(9.3,4.6)$ & $2,3,3 a, 9^{\prime}$ & $46.9, \mathrm{CH}$ & $4.83, \mathrm{q}(6.2)$ & $2,3,3 a, 9$ \\
\hline 9 & $41.1, \mathrm{CH}_{2}$ & $\begin{array}{c}3.49, \mathrm{~m} \\
3.36, \text { under } \mathrm{H}_{2} \mathrm{O}\end{array}$ & & $42.1, \mathrm{CH}_{2}$ & $\begin{array}{l}\text { 3.83, dt }(13.8,7.2) \\
3.70, \mathrm{dt}(13.8,5.7)\end{array}$ & $3,8,9^{\prime}$ \\
\hline 10-NH & & 7.98, br s & & & $8.95, \mathrm{t}(6.1)$ & $9,9^{\prime}$ \\
\hline $1^{\prime}$ & & $12.27, \mathrm{~d}(3.2)$ & $2^{\prime}, 3^{\prime}$ & & $12.26, \mathrm{~d}(3.0)$ & $2^{\prime}, 3^{\prime}, 3^{\prime} \mathrm{a}, 7^{\prime} \mathrm{a}$ \\
\hline $2^{\prime}$ & 138.6, $\mathrm{CH}$ & $8.80, \mathrm{~d}(3.2)$ & $3^{\prime}, 3^{\prime} \mathrm{a}, 7^{\prime} \mathrm{a}$ & 138.7, CH & $8.77, \mathrm{~d}(3.3)$ & $3^{\prime}, 3^{\prime} a, 7^{\prime} a$ \\
\hline $3^{\prime}$ & $112.1, \mathrm{C}$ & & & $112.1, \mathrm{C}$ & & \\
\hline $3^{\prime} \mathrm{a}$ & 126.3, C & & & $126.3, \mathrm{C}$ & & \\
\hline $4^{\prime}$ & 121.2, $\mathrm{CH}$ & $8.22, \mathrm{dd}(7.2,2.1)$ & $6^{\prime}, 7^{\prime} \mathrm{a}$ & 121.3, $\mathrm{CH}$ & $8.22, \mathrm{dd}(6.8,2.3)$ & $6^{\prime}, 7^{\prime} \mathrm{a}$ \\
\hline $5^{\prime}$ & $122.6, \mathrm{CH}$ & $7.23, \operatorname{td}(7.2,2.0)$ & $3^{\prime} \mathrm{a}, 7^{\prime}$ & $122.7, \mathrm{CH}$ & $7.25, \operatorname{td}(6.9,2.2)$ & $3^{\prime} \mathrm{a}, 7^{\prime}$ \\
\hline $6^{\prime}$ & 123.5, $\mathrm{CH}$ & $7.25, \mathrm{td}(7.2,2.0)$ & $4^{\prime}, 7^{\prime} \mathrm{a}$ & 123.6, $\mathrm{CH}$ & $7.27, \operatorname{td}(6.9,2.3)$ & $4^{\prime}, 7^{\prime} \mathrm{a}$ \\
\hline $7^{\prime}$ & $112.6, \mathrm{CH}$ & $7.53, \mathrm{dd}(7.2,2.0)$ & $3^{\prime} \mathrm{a}, 5^{\prime}$ & $112.7, \mathrm{CH}$ & $7.54, \mathrm{dd}(7.0,2.2)$ & $3^{\prime} a, 5^{\prime}$ \\
\hline $7^{\prime} \mathrm{a}$ & $136.2, \mathrm{C}$ & & & $136.2, \mathrm{C}$ & & \\
\hline $8^{\prime}$ & 181.2, C & & & $180.9, \mathrm{C}$ & & \\
\hline $9^{\prime}$ & $163.1, \mathrm{C}$ & & & 163.7, C & & \\
\hline $10^{\prime}-\mathrm{NH}$ & & $9.15, \mathrm{~d}(9.2)$ & $8,9^{\prime}$ & & 8.33, br d (4.3) & 3 \\
\hline
\end{tabular}




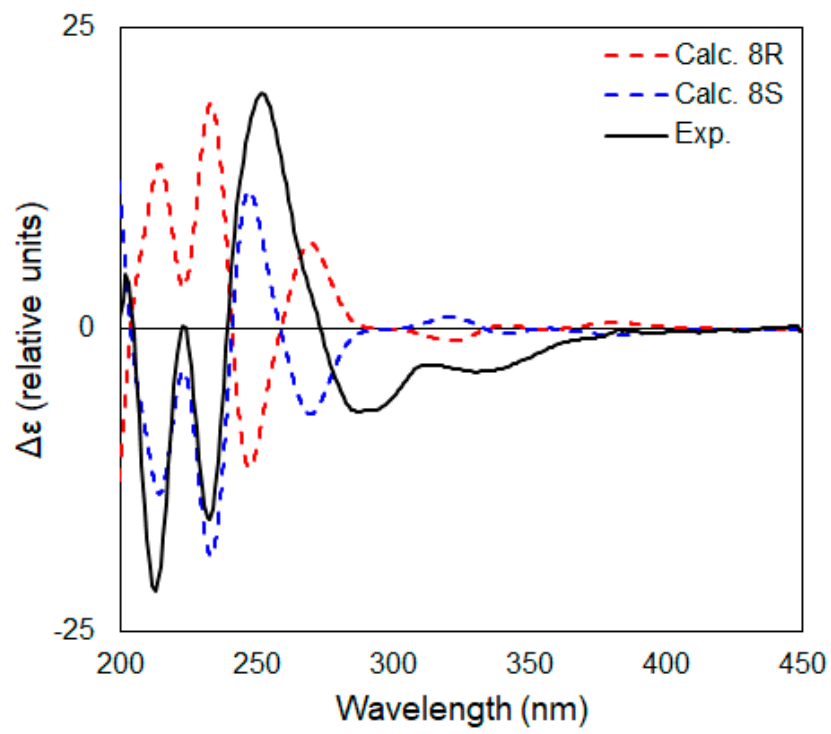

Figure 2. Comparison between the two TDDFT predicted electronic circular dichroism (ECD) spectra of both enantiomers of $\mathbf{1}$ and the experimental spectrum of $\mathbf{1}$.

Calcicamides (1-2) differ from other bisindole compounds due to the lack of a central heterocyclic ring, which is instead opened in $\mathbf{1}$ and $\mathbf{2}$. As the tryptophan origin of bisindole alkaloids cannot be contested, we hypothesize that the nucleophilicity of one primary amine should enable the connection between two 8-ketotryptamine derivative units after a first condensation (Scheme 1) [3]. Hydration/oxidation of the intermediate would lead to compound 1. Oxidation of the 8-ketotryptamine derivatives at C-9 prior to isomerisation would lead to the monoindole derivatives 7 and 8 . The piperazine central core of hamacanthins and dragmacidins would subsequently be formed when the second condensation occurs at C-8' of compound $\mathbf{1}$ or $\mathbf{2}$. To assess if calcicamides are natural products or hydrolysed artefacts produced during the isolation process, the extracts and fractions were analysed by LC-MS/MS prior to acidic HPLC isolation. We identified two peaks in the chromatogram with a molecular ion at $\mathrm{m} / \mathrm{z}$ 425/427 (1:1) coeluting with the two isomers of calcicamides. This result came as a proof of their natural origin. Additionally, coscinamides isolated from the marine sponge Coscinoderma sp. are another family of bisindole alkaloids that lack a central ring and instead contain a linear chain with an alpha keto enamide [15]. We hypothesise that the coscinamides may also be biosynthetically related to calcicamide B (2) following a deamination at C-8.

Members of the bisindole alkaloid family have been previously reported with a large array of bioactivities including moderately potent antiviral, antitumoral, antibacterial, and antifungal activities $[5,10,16]$. The six bisindole alkaloids were screened for their antitumoral activity against a HeLa cell line. Compounds $\mathbf{2}$ and $\mathbf{4}-\mathbf{6}$ exhibited weak activity against these cells with $\mathrm{IC}_{50} \mathrm{~s}$ between 100 and $200 \mu \mathrm{M}$ (Table S1). Compounds 1 and 3 did not induce detectable cell lysis at the highest tested concentrations (185 and $166 \mu \mathrm{M}$ respectively). Most cell death occurred within the first $6 \mathrm{~h}$ of incubation. Cell lysis increased between 6 and $24 \mathrm{~h}$ incubation, corresponding to an increase in the cytotoxicity with longer exposure. Compounds 3-6 have previously been shown to have moderate cytotoxicity against adenocarcinoma (AGS) and lymphocytic leukaemia (L1210) cells. This previous study noted that the cytotoxicity of Spongosorites bisindoles is cell-line dependent with only weak activity reported against other cell lines screened [10]. The inability of these compounds to display comparable activity against HeLa cells again suggests that these compounds may be more specific against carcinoma and leukaemia cell lines. Therefore, based on these results we will continue a deeper evaluation of the cytotoxicity of Spongosorites bisindoles against carcinoma and leukaemia cell lines. 


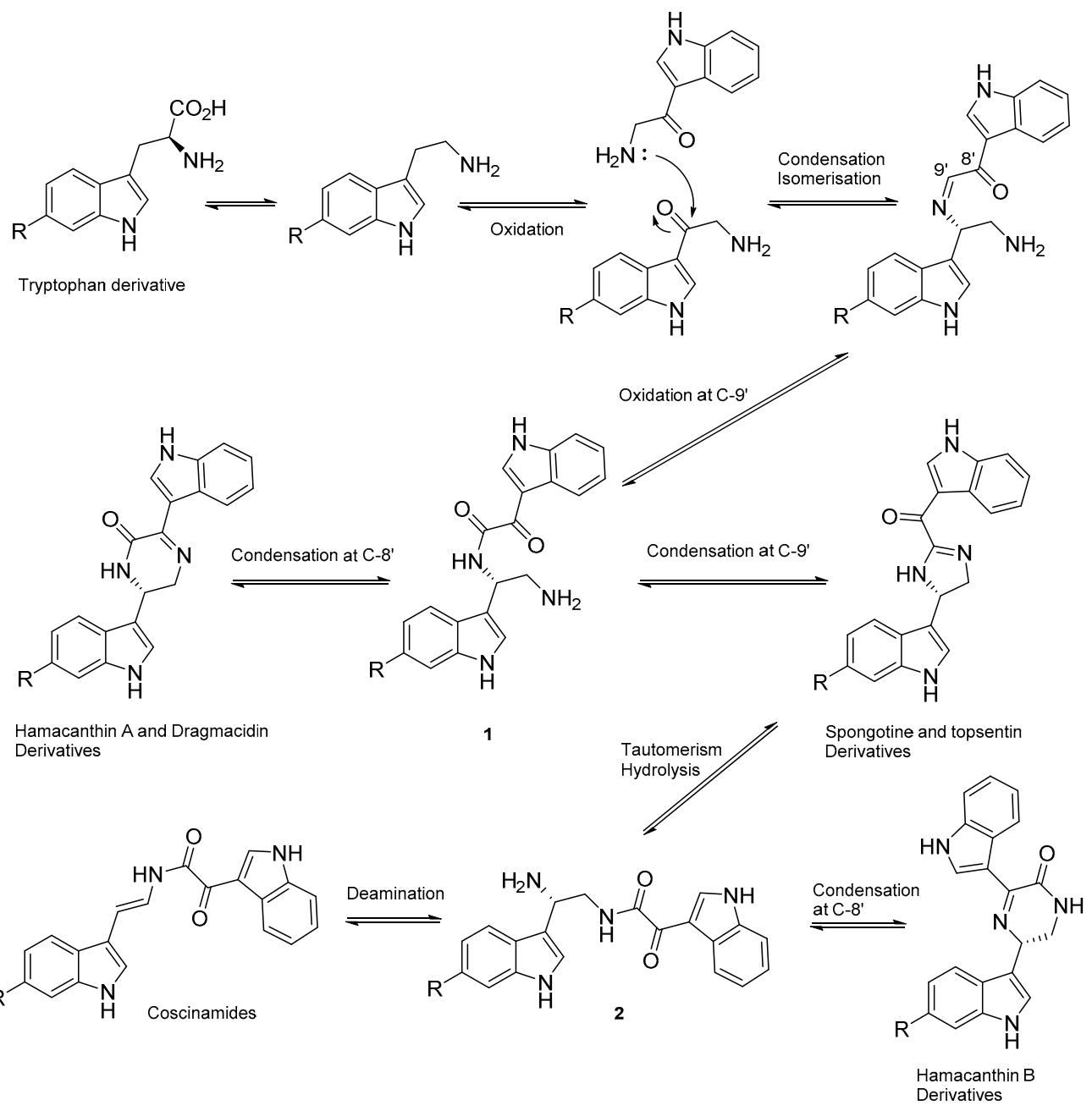

Scheme 1. Biosynthetic hypothesis for the calcicamide, hamacanthin, coscinamides, and topsentin derivatives.

\section{Materials and Methods}

\subsection{General Experimental Procedures}

Optical rotations were recorded on a Unipol L1000 polarimeter at the sodium D-line $(589.3 \mathrm{~nm})$ with a $5 \mathrm{~cm}$ cell at $20{ }^{\circ} \mathrm{C}$ (Schmidt+Haensch, Berlin, Germany). UV and ECD were recorded on a Chirascan V100 with a $1.0 \mathrm{~cm}$ quartz cuvette (Applied Photophysics, Leatherhead, UK). IR data were recorded on a PerkinElmer spectrum 100 FT-IR spectrometer (PerkinElmer, Waltham, MA, USA). NMR experiments were performed on a $500 \mathrm{MHz}$ Varian Inova spectrometer (Agilent, Santa Clara, CA, USA). Chemical shifts $\left(\delta\right.$ in ppm) were referenced to the carbon $\left(\delta_{\mathrm{C}} 39.52\right)$ and proton $\left(\delta_{\mathrm{H}} 2.50\right)$ signals of DMSO- $d_{6}$. HRESIMS were obtained using an Agilent 6540 Q-Tof mass spectrometer equipped with an Agilent 1290 UPLC and autosampler (Agilent). Preparative and semipreparative HPLC was carried out on a Jasco LC-2000 series equipped with a coupled UV detector. Analytical HPLC was carried out on an Agilent 1260 HPLC system equipped with a DAD detector coupled with an Agilent 385-ELSD. All solvents used for extraction and separation were HPLC grade, and $\mathrm{H}_{2} \mathrm{O}$ was Milli-Q (Millipore Ireland B.V., Carrigtwohill, County Cork, Ireland) filtered.

\subsection{Biological Material}

Spongosorites calcicola was collected at Rathlin Island (Co. Antrim, Northern Ireland) at $18 \mathrm{~m}$ depth by SCUBA. The sponge was taxonomically identified by Christine Morrow through morphological 
and spicule analysis. A voucher specimen of this sample "BDV10015" is kept at the National Marine Biodiscovery Laboratory (Marine Institute, Oranmore, Co. Galway, Ireland).

\subsection{Extraction and Isolation}

The freeze-dried sponge material $(5.20 \mathrm{~g})$ was extracted with a solvent mixture of $1: 1(\mathrm{v} / \mathrm{v})$ $\mathrm{MeOH} / \mathrm{CH}_{2} \mathrm{Cl}_{2}$ under sonication to yield an extract $(970 \mathrm{mg})$. The extract was then fractionated using SPE on $\mathrm{C}_{18}$-bonded silica gel, eluting with varying solvent mixtures to yield five fractions: $100 \%$ $\mathrm{H}_{2} \mathrm{O}, 50 \% \mathrm{H}_{2} \mathrm{O} / 50 \% \mathrm{MeOH}\left(331.9 \mathrm{mg}\right.$ ), 25\% $\mathrm{H}_{2} \mathrm{O} / 75 \% \mathrm{MeOH}(115.7 \mathrm{mg}$ ), 100\% MeOH (67.8 mg), 50\% $\mathrm{MeOH} / 50 \% \mathrm{CH}_{2} \mathrm{Cl}_{2}(81.5 \mathrm{mg})$. The $75 \%$ and $100 \% \mathrm{MeOH}$ fractions with similar profiles were then combined for HPLC purification. The resulting fraction was separated using preparative HPLC on a phenyl-hexyl column (Waters, Xselect CSH phenyl-hexyl $130 \AA$ A, $5 \mu \mathrm{m}, 19 \times 250 \mathrm{~mm}$ ). The column was first eluted with $90 \% \mathrm{H}_{2} \mathrm{O}(0.1 \% \mathrm{TFA}) / 10 \% \mathrm{MeOH}(0.1 \%$ TFA) for $5 \mathrm{~min}$, followed by a linear gradient to $100 \% \mathrm{MeOH}(0.1 \% \mathrm{TFA})$ over $20 \mathrm{~min}$, and the column was then further eluted with the final conditions for $7 \mathrm{~min}$, all at a flow rate of $10 \mathrm{~mL} / \mathrm{min}$. This resulted in the collection of six peaks from the UV chromatogram; these peaks were dried and re-purified using semi-preparative and analytical HPLC.

Peak $2(11.63 \mathrm{mg})$ was re-purified on a $\mathrm{C}_{18}$ analytical column (Macherey-Nagel Nucleodur HTec $\left.\mathrm{C}_{18}, 5 \mu \mathrm{m}, 4.6 \times 250 \mathrm{~mm}\right)$ eluting first with $74 \% \mathrm{H}_{2} \mathrm{O}(0.1 \% \mathrm{TFA}) / 26 \% \mathrm{MeOH}(0.1 \% \mathrm{TFA})$ for $5 \mathrm{~min}$, followed by a linear gradient to $50 \% \mathrm{H}_{2} \mathrm{O}(0.1 \%$ TFA $) / 50 \% \mathrm{MeOH}(0.1 \%$ TFA) over $20 \mathrm{~min}$, another gradient to $100 \% \mathrm{MeOH}(0.1 \%$ TFA) over $10 \mathrm{~min}$ was performed and the column was then further eluted with the final conditions for $7 \mathrm{~min}$, all at a flow rate of $1.0 \mathrm{~mL} / \mathrm{min}$. This yielded the new compound calcicamide A $(\mathbf{1}, 6.24 \mathrm{mg}, 0.12 \%$ dry wt.) and the known compound trans-3,4-dihydrohamacanthin A $\left(3,3.11 \mathrm{mg}, 6.0 \times 10^{-2} \%\right.$ dry wt.).

Peaks 3 and 4 were combined $\left(82.74 \mathrm{mg}\right.$ ) and re-purified on a $\mathrm{C}_{18}$ semiprep column (Waters symmetry C18 prep, $7 \mu \mathrm{m}, 7.8 \times 250 \mathrm{~mm})$ eluting with isocratic conditions of $62 \% \mathrm{H}_{2} \mathrm{O}(0.1 \%$ TFA $) / 38 \%$ $\mathrm{ACN}(0.1 \% \mathrm{TFA})$ for $25 \mathrm{~min}$ at a flow rate of $4.0 \mathrm{~mL} / \mathrm{min}$. This yielded the known compound spongotine A $(6,39.03 \mathrm{mg}, 0.75 \%$ dry wt.), the new compound calcicamide B $(2,7.8 \mathrm{mg}, 0.15 \%$ dry wt. $)$, and the known compounds bromotopsentin $(5,42.12 \mathrm{mg}, 0.81 \%$ dry wt.) and 2-(1H-indol-3-yl)-2-oxoacetate methyl ester $\left(7,1.4 \mathrm{mg}, 2.6 \times 10^{-2} \%\right.$ dry wt.).

Peak $5\left(39.24 \mathrm{mg}\right.$ ) was re-purified on a $\mathrm{C}_{18}$ analytical column (Macherey-Nagel Nucleodur HTec $\left.\mathrm{C}_{18}, 5 \mu \mathrm{m}, 4.6 \times 250 \mathrm{~mm}\right)$ eluting first with $90 \% \mathrm{H}_{2} \mathrm{O}(0.1 \%$ TFA $) / 10 \% \mathrm{MeOH}(0.1 \%$ TFA) for $5 \mathrm{~min}$, followed by a linear gradient to $100 \% \mathrm{MeOH}(0.1 \%$ TFA) over $20 \mathrm{~min}$, and the column was further eluted with the final conditions for $10 \mathrm{~min}$, all at a flow rate of $1.2 \mathrm{~mL} / \mathrm{min}$. This yielded the known compound bromodeoxytopsentin $(4,37.96 \mathrm{mg}, 0.73 \%$ dry wt.). Peak 6 (24.02 mg) was re-purified with the same column and conditions used for peak 5 . This yielded the known compound 2-(6-bromo-1H-indol-3-yl)-2-oxoacetate methyl ester (8, $1.0 \mathrm{mg}, 1.9 \times 10^{-2} \%$ dry wt.).

Calcicamide A (1): Bright yellow, amorphous solid; $[\alpha]_{\mathrm{D}}^{20}+0.3\left(c 0.1, \mathrm{CH}_{3} \mathrm{CN}\right)$; UV/Vis $\left(c 1 \times 10^{-4} \mathrm{M}\right.$, $\left.\mathrm{CH}_{3} \mathrm{CN}\right) \lambda_{\max }(\log \varepsilon) 329(3.50), 293$ (3.42), 280 (3.63), 245 (3.63), $213(4.14) \mathrm{nm} ; \mathrm{ECD}\left(c 1 \times 10^{-4} \mathrm{M}_{,} \mathrm{CH}_{3} \mathrm{CN}\right)$ $\lambda_{\max }(\Delta \varepsilon) 331(-0.2), 287(-0.37), 252(+1.06), 233(-0.86), 213(-1.18) \mathrm{nm} ;{ }^{1} \mathrm{H}-\mathrm{NMR}$ (DMSO- $\left.d_{6}, 500 \mathrm{MHz}\right)$ and ${ }^{13} \mathrm{C}-\mathrm{NMR}$ data see Table 1; HRESIMS (+) $\mathrm{m} / \mathrm{z} 425.0614[\mathrm{M}+\mathrm{H}]^{+}$(calcd. for $\mathrm{C}_{20} \mathrm{H}_{18}{ }^{79} \mathrm{BrN}_{4} \mathrm{O}_{2}{ }^{+}$, 425.0608, $\Delta+1.4 \mathrm{ppm})$.

Calcicamide B (2): Bright yellow, amorphous solid; $[\alpha]_{\mathrm{D}}^{20}+0.2\left(c 0.1, \mathrm{CH}_{3} \mathrm{CN}\right)$; UV/Vis $\left(c 5 \times 10^{-5} \mathrm{M}\right.$, $\left.\mathrm{CH}_{3} \mathrm{CN}\right) \lambda_{\max }(\log \varepsilon) 314$ (3.23), 239 (3.40), $210(3.87) \mathrm{nm} ; \mathrm{ECD}\left(c 5 \times 10^{-5} \mathrm{M}, \mathrm{CH}_{3} \mathrm{CN}\right) \lambda_{\max }(\Delta \varepsilon) 335(-0.93)$, $272(-1.48), 244(-1.39), 231(+0.63), 218(-0.13) \mathrm{nm} ;{ }^{1} \mathrm{H}-\mathrm{NMR}$ (DMSO- $d_{6}, 500 \mathrm{MHz}$ ) and ${ }^{13} \mathrm{C}-\mathrm{NMR}$ data see Table 1; HRESIMS (+) $\mathrm{m} / \mathrm{z} 425.0608[\mathrm{M}+\mathrm{H}]^{+}$(calcd. for $\mathrm{C}_{20} \mathrm{H}_{18}{ }^{79} \mathrm{BrN}_{4} \mathrm{O}_{2}{ }^{+}, 425.0608, \Delta+0.0 \mathrm{ppm}$ ).

trans-3,4-Dihydrohamacanthin $A$ (3) [13]: Yellow, amorphous solid; ${ }^{1} \mathrm{H}-\mathrm{NMR}$ (DMSO- $d_{6}, 500 \mathrm{MHz}$ ) $\delta$ $11.54(1 \mathrm{H}, \mathrm{s}), 11.48(1 \mathrm{H}, \mathrm{s}), 10.06(1 \mathrm{H}, \mathrm{brd}, J=9.7 \mathrm{~Hz}), 9.83(1 \mathrm{H}, \mathrm{brq}, J=9.4 \mathrm{~Hz}), 8.72(1 \mathrm{H}, \mathrm{d}, J=2.6 \mathrm{~Hz})$, $7.82(1 \mathrm{H}, \mathrm{d}, J=8.6 \mathrm{~Hz}), 7.67(1 \mathrm{H}, \mathrm{s}), 7.63(1 \mathrm{H}, \mathrm{d}, J=1.7 \mathrm{~Hz}), 7.62(1 \mathrm{H}, \mathrm{d}, J=1.7 \mathrm{~Hz}), 7.54(1 \mathrm{H}, \mathrm{s}), 7.53$ $(1 \mathrm{H}, \mathrm{d}, J=8.6 \mathrm{~Hz}), 7.27(1 \mathrm{H}, \mathrm{dd}, J=8.6,1.7 \mathrm{~Hz}), 7.23(1 \mathrm{H}, \mathrm{dd}, J=8.6,1.7 \mathrm{~Hz}), 5.71(1 \mathrm{H}, \mathrm{m}), 5.37(1 \mathrm{H}, \mathrm{m})$, $4.09(1 \mathrm{H}, \mathrm{m}), 3.64(1 \mathrm{H}, \mathrm{m})$ and ${ }^{1} \mathrm{H}-\mathrm{NMR}\left(\mathrm{MeOH}-d_{4}, 500 \mathrm{MHz}\right) \delta 7.77(1 \mathrm{H}, \mathrm{d}, J=8.6 \mathrm{~Hz}), 7.61(1 \mathrm{H}, \mathrm{d}$, 
$J=1.7 \mathrm{~Hz}), 7.61(1 \mathrm{H}, \mathrm{d}, J=1.7 \mathrm{~Hz}), 7.58(1 \mathrm{H}, \mathrm{s}), 7.56(1 \mathrm{H}, \mathrm{d}, J=8.6 \mathrm{~Hz}), 7.52(1 \mathrm{H}, \mathrm{s}), 7.30(1 \mathrm{H}, \mathrm{dd}, J=8.6$, $1.7 \mathrm{~Hz}), 7.26(1 \mathrm{H}, \mathrm{dd}, J=8.6,1.7 \mathrm{~Hz}), 5.80(1 \mathrm{H}, \mathrm{s}), 5.37(1 \mathrm{H}, \mathrm{m}), 4.09(1 \mathrm{H}, \mathrm{m}), 3.64(1 \mathrm{H}, \mathrm{m}) ; \operatorname{HRESIMS}(+)$ $m / z$ 486.9769/488.9747/490.9726 (1:2:1) $[\mathrm{M}+\mathrm{H}]^{+}$(calcd. for $\mathrm{C}_{20} \mathrm{H}_{17}{ }^{79} \mathrm{Br}_{2} \mathrm{~N}_{4} \mathrm{O}^{+}, 486.9764, \Delta+1.0 \mathrm{ppm}$ ).

Bromodeoxytopsentin (4) [13,14]: Yellow, amorphous solid; ${ }^{1} \mathrm{H}-\mathrm{NMR}$ (DMSO- $\left.d_{6}, 500 \mathrm{MHz}\right) \delta 12.13(1 \mathrm{H}$, brs), $11.51(1 \mathrm{H}, \mathrm{brs}), 9.20(1 \mathrm{H}, \mathrm{s}), 8.36(1 \mathrm{H}, \mathrm{brd}, J=7.3 \mathrm{~Hz}), 7.98(1 \mathrm{H}, \mathrm{d}, J=8.4 \mathrm{~Hz}), 7.94(1 \mathrm{H}, \mathrm{s}), 7.68$ $(1 \mathrm{H}, \mathrm{s}), 7.64(1 \mathrm{H}, \mathrm{s}), 7.54(1 \mathrm{H}, \mathrm{brd}, J=7.0 \mathrm{~Hz}), 7.24(3 \mathrm{H}, \mathrm{m})$ and ${ }^{13} \mathrm{C}-\mathrm{NMR}$ data (DMSO- $\left.d_{6}, 125 \mathrm{MHz}\right) \delta$ 175.9, 145.2, 137.3, 136.9, 136.2, 126.7, 124.3 (br), 123.8, 122.9, 122.4, 121.9, 121.7, 121.6, 114.4, 114.3, 113.7, 112.3 (NOTE: some carbons could not be observed); HRESIMS (+) $\mathrm{m} / \mathrm{z}$ 405.0352/407.0330 (1:1) $[\mathrm{M}+\mathrm{H}]^{+}$ (calcd. for $\mathrm{C}_{20} \mathrm{H}_{14}{ }^{79} \mathrm{BrN}_{4} \mathrm{O}^{+}, 405.0346, \Delta+1.5 \mathrm{ppm}$ ).

Bromotopsentin (5) [13,14]: Yellow, amorphous solid; ${ }^{1} \mathrm{H}-\mathrm{NMR}$ (DMSO- $\left.d_{6}, 500 \mathrm{MHz}\right) \delta 11.83(1 \mathrm{H}, \mathrm{d}$, $J=2.4 \mathrm{~Hz}), 11.53(1 \mathrm{H}, \mathrm{brs}), 8.99(1 \mathrm{H}, \mathrm{d}, J=2.4 \mathrm{~Hz}), 8.14(1 \mathrm{H}, \mathrm{d}, J=8.6 \mathrm{~Hz}), 8.00(1 \mathrm{H}, \mathrm{d}, J=8.5 \mathrm{~Hz})$, $7.99(1 \mathrm{H}, \mathrm{d}, J=2.2 \mathrm{~Hz}), 7.73(1 \mathrm{H}, \mathrm{s}), 7.66(1 \mathrm{H}, \mathrm{d}, J=1.7 \mathrm{~Hz}), 7.25(1 \mathrm{H}, \mathrm{dd}, J=8.5,1.7 \mathrm{~Hz}), 6.91(1 \mathrm{H}$, $\mathrm{d}, J=2.0 \mathrm{~Hz}), 6.77(1 \mathrm{H}, \mathrm{dd}, J=8.6,2.0 \mathrm{~Hz})$ and ${ }^{13} \mathrm{C}-\mathrm{NMR}$ data (DMSO- $\left.d_{6}, 125 \mathrm{MHz}\right) \delta 175.2,154.4$, 144.8, 137.6, 137.4, 136.0, 133.5 (br), 124.6, 123.7, 122.5, 122.1, 121.6, 119.5, 119.1 (br), 114.5, 114.4, 113.9, 112.2, 107.5 (br), 97.6; HRESIMS (+) $m / z$ 421.0302/423.0280 (1:1) $[\mathrm{M}+\mathrm{H}]^{+}$(calcd. for $\mathrm{C}_{20} \mathrm{H}_{14}{ }^{79} \mathrm{BrN}_{4} \mathrm{O}_{2}{ }^{+}$, 421.0295, $\Delta+1.7 \mathrm{ppm})$.

Spongotine A (6) [5]: Yellow, amorphous solid; $[\alpha]_{20}{ }^{\mathrm{D}}-9.2$ (c 1.0, ACN); ${ }^{1} \mathrm{H}-\mathrm{NMR}$ (DMSO- $d_{6}, 500 \mathrm{MHz}$ ) $\delta 12.86(1 \mathrm{H}, \mathrm{s}), 11.50(1 \mathrm{H}, \mathrm{s}), 11.34(1 \mathrm{H}, \mathrm{brs}), 11.14(1 \mathrm{H}, \mathrm{s}), 8.57(1 \mathrm{H}, \mathrm{d}, J=3.5 \mathrm{~Hz}), 8.18(1 \mathrm{H}, \mathrm{dd}, J=7.0$, $2.1 \mathrm{~Hz}), 7.67(1 \mathrm{H}, \mathrm{d}, J=1.7 \mathrm{~Hz}), 7.66(1 \mathrm{H}, \mathrm{d}, J=2.5 \mathrm{~Hz}), 7.62(1 \mathrm{H}, \mathrm{dd}, J=7.0,1.8 \mathrm{~Hz}), 7.56(1 \mathrm{H}, \mathrm{d}$, $J=8.5 \mathrm{~Hz}), 7.38(1 \mathrm{H}, \mathrm{td}, J=7.0,2.1 \mathrm{~Hz}), 7.36(1 \mathrm{H}, \mathrm{td}, J=7.0,1.8 \mathrm{~Hz}), 7.25(1 \mathrm{H}, \mathrm{dd}, J=8.5,1.7 \mathrm{~Hz}), 5.87$ $(1 \mathrm{H}, \mathrm{dd}, J=12.2,9.3 \mathrm{~Hz}), 4.52(1 \mathrm{H}, \mathrm{t}, J=12.0 \mathrm{~Hz}), 4.08(1 \mathrm{H}, \mathrm{dd}, J=11.6,9.3 \mathrm{~Hz})$ and ${ }^{13} \mathrm{C}-\mathrm{NMR}$ data (DMSO- $\left.d_{6}, 125 \mathrm{MHz}\right) \delta 173.1,161.7,140.1,137.6,137.3,125.9,125.0,124.7,124.0,123.7,122.3,121.2$, $120.1,114.8,114.7,113.4,113.3,112.6,54.4,51.3$; HRESIMS (+) $\mathrm{m} / \mathrm{z}$ 407.0512/409.0489 $(1: 1)[\mathrm{M}+\mathrm{H}]^{+}$ (calcd. for $\mathrm{C}_{20} \mathrm{H}_{16}{ }^{79} \mathrm{BrN}_{4} \mathrm{O}^{+}, 407.0502, \Delta+2.5 \mathrm{ppm}$ ).

2-(1H-Indol-3-yl)-2-oxoacetate methyl ester (7) [3]: Yellow, amorphous solid; ${ }^{1} \mathrm{H}-\mathrm{NMR}\left(\mathrm{CD}_{3} \mathrm{OD}-d_{4}\right.$, $500 \mathrm{MHz}) \delta 12.47(1 \mathrm{H}, \mathrm{s}), 8.47(1 \mathrm{H}, \mathrm{d}, J=3.3 \mathrm{~Hz}),, 8.08(1 \mathrm{H}, \mathrm{d}, J=8.5 \mathrm{~Hz}),, 7.73(1 \mathrm{H}, \mathrm{d}, J=1.7 \mathrm{~Hz}),$, $(1 \mathrm{H}, \mathrm{dd}, J=8.5,1.8 \mathrm{~Hz}),, 3.88(3 \mathrm{H}, \mathrm{s})$; HRESIMS $(+) \mathrm{m} / \mathrm{z} 204.0665[\mathrm{M}+\mathrm{H}]^{+}$(calcd. for $\mathrm{C}_{11} \mathrm{H}_{10} \mathrm{NO}_{3}{ }^{+}$, 204.0655, $\Delta+4.9 \mathrm{ppm})$.

2-(6-Bromo-1H-indol-3-yl)-2-oxoacetate methyl ester (8) [3]: Yellow, amorphous solid; ${ }^{1} \mathrm{H}-\mathrm{NMR}$ (DMSO- $d_{6}$, $500 \mathrm{MHz}) \delta 12.47(1 \mathrm{H}, \mathrm{s}), 8.47(1 \mathrm{H}, \mathrm{d}, J=3.3 \mathrm{~Hz}),, 8.08(1 \mathrm{H}, \mathrm{d}, J=8.5 \mathrm{~Hz}),, 7.73(1 \mathrm{H}, \mathrm{d}, J=1.7 \mathrm{~Hz}),$, $(1 \mathrm{H}, \mathrm{dd}, J=8.5,1.8 \mathrm{~Hz}),, 3.88(3 \mathrm{H}, \mathrm{s})$; HRESIMS $(+) \mathrm{m} / \mathrm{z} 281.9763 / 283.9745(1: 1)[\mathrm{M}+\mathrm{H}]^{+}$(calcd. for $^{2}$ $\left.\mathrm{C}_{11} \mathrm{H}_{9}{ }^{79} \mathrm{BrNO}_{3}{ }^{+}, 281.9760, \Delta+1.1 \mathrm{ppm}\right)$.

\subsection{Computational Methods}

Conformational analyses of each compound were performed with Schrodinger MacroModel [17]. These conformers were then optimized using DFT, at the B3LYP/6-311+G(2d,p) level in Gaussian 16 . At the same time, the zero-point energy, electronic transition, and rational strength of all conformers were calculated. Following this the ECD spectra for each conformer were calculated in Gaussian 16 at the B3LYP/6-311G(2d,p) level [18]. All DFT calculations were performed using a polarisable continuum model [19]. The final ECD spectra were produced using the freely available software SpecDis 1.7 (version 1.71, SpecDis, Berlin, Germany) and corrected with the experimental UV spectra [20].

\subsection{Biological Screening}

HeLa cells were cultured in DMEM complete (Dulbecco's Minimal Eagles Medium-low glucose and without phenol red containing $10 \%(v / v)$ foetal bovine serum (FBS), non-essential amino acids, $20 \mathrm{mmol} \mathrm{L}$-glutamine, $100 \mu \mathrm{g} \cdot \mathrm{mL}^{-1}$ penicillin and $100 \mu \mathrm{g} \cdot \mathrm{mL}^{-1}$ streptomycin) at $37^{\circ} \mathrm{C}, 5 \% \mathrm{CO}_{2}$. For co-incubation experiments, cells were seeded at 20,000 cells per mL DMEM complete per well in 48 -well tissue culture plates. After $16 \mathrm{~h}$, monolayers were washed with PBS and $500 \mu \mathrm{L}$ DMEM 
was added per well with the sponge fractions $(100 \mu \mathrm{g} / \mathrm{mL})$ or isolated molecules $(12-200 \mu \mathrm{M})$. Non-treated cells acted as the $0 \%$ lysis control and cells treated with $0.8 \%$ Triton X-100 were the 100\% lysis control. After 6 and 24 h, cytotoxicity was determined using the Promega CytoTox 96 Non-Radioactive Cytotoxicity Assay kit, according to the manufacturer's directions, to measure lactate dehydrogenase released from lysed cells. Data are presented as means \pm SD of three independent experiments performed.

\section{Conclusions}

The first chemical investigation of the sponge Spongosorites calcicola collected in the Celtic Sea led to the isolation of two new bisindole alkaloids that are likely important biosynthetic intermediates in the metabolic pathway to known analogues. Bisindole alkaloids represent an important class of sponge natural products for pharmaceutical applications, and our study highlighted some selectivity for cytotoxicity.

Supplementary Materials: Electronic supplementary information including MS, NMR, IR and ECD spectra of new compounds is available online at http://www.mdpi.com/1420-3049/24/21/3890/s1.

Author Contributions: Conceptualization, A.B. and O.P.T.; methodology, L.K.J., N.M.D.K., N.K. and D.R.; formal analysis, L.K.J., N.M.D.K., N.K., D.R. and C.M.; writing-original draft preparation, L.K.J. and N.M.D.K.; writing-review and editing, A.B. and O.P.T.; project administration, O.P.T.; funding acquisition, A.B. and O.P.T.

Funding: This research was funded by the Marine Institute under the Marine Research Program by the Irish Government (Grant-Aid Agreement No. PBA/MB/16/01). This project has also received funding under the Irish Research Council GOIPG/2017/910 (N.M.D.K.).

Acknowledgments: We thank R. Doohan (NUI Galway) for her help with the NMR analysis. The authors wish to acknowledge the Irish Centre for High-End Computing (ICHEC) for the provision of computational facilities and support.

Conflicts of Interest: The authors declare no conflict of interest.

\section{References}

1. Blunt, J.W.; Copp, B.R.; Hu, W.; Munro, M.H.G.; Northcote, P.T.; Prinsep, M.R. Marine natural products. Nat. Prod. Rep. 2008, 36, 35-94. [CrossRef] [PubMed]

2. Carroll, A.R.; Copp, B.R.; Davis, R.A.; Keyzers, R.A.; Prinsep, M.R. Marine natural products. Nat. Prod. Rep. 2019, 36, 122-173. [CrossRef] [PubMed]

3. Bao, B.; Zhang, P.; Lee, Y.; Hong, J.; Lee, C.; Jung, J.H. Monoindole Alkaloids from a Marine Sponge Spongosorites sp. Mar. Drugs 2007, 5, 31-39. [CrossRef] [PubMed]

4. Bao, B.; Sun, Q.; Yao, X.; Hong, J.; Lee, C.; Sim, C.J.; Im, K.S.; Jung, J.H. Cytotoxic Bisindole Alkaloids from a Marine Sponge Spongosorites sp. J. Nat. Prod. 2005, 68, 711-715. [CrossRef] [PubMed]

5. Bao, B.; Sun, Q.; Yao, X.; Hong., J.; Lee, C.; Cho, H.Y.; Jung, J.H. Bisindole Alkaloids of the Topsentin and Hamacanthin Classes from a Marine Sponge Spongosorites sp. J. Nat. Prod. 2007, 70, 2-8. [CrossRef] [PubMed]

6. Bartik, K.; Braekman, J.; Daloze, D.; Stoller, C. Topsentins, new toxic bis-indole alkaloids from the marine sponge Topsentia genitrix. Can. J. Chem. 1987, 65, 2118-2121. [CrossRef]

7. Wright, A.E.; Killday, K.B.; Chakrabarti, D.; Guzmán, E.A.; Harmody, D.; McCarthy, P.J.; Pitts, T.; Pomponi, S.A.; Reed, J.K.; Roberts, B.F.; et al. A Bioactive Bis-Indole Alkaloid from a Deep-Water Sponge of the Genus Spongosorites. Mar. Drugs 2017, 15, 16. [CrossRef] [PubMed]

8. Gunasekera, S.P.; McCarthy, P.J.; Kelly-Borges, M. Hamacanthins A and B, new antifungal bis indole alkaloids from the deep-water marine sponge, Hamacantha sp. J. Nat. Prod. 1994, 57, 1437-1441. [CrossRef] [PubMed]

9. Kohmoto, S.; Kashman, Y.; McConnell, O.J.; Rinehart, K.L.J.; Wright, A.; Koehn, F. Dragmacidin, a new cytotoxic bis(indole) alkaloid from a deep water marine sponge, Dragmacidon sp. J. Org. Chem. 1988, 53, 3116-3118. [CrossRef]

10. Oh, K.; Mar, W.; Kim, S.; Kim, J.; Lee, T.; Kim, J.; Shin, D.; Sim, C.J.; Shin, J. Antimicrobial activity and cytotoxicity of bis (indole) alkaloids from the sponge Spongosorites sp. Biol. Pharm. Bull. 2006, 29, 570-573. [CrossRef] [PubMed] 
11. Oh, K.; Mar, W.; Kim, S.; Kim, J.; Kim, J.; Oh, M.; Kim, J.; Shin, D.; Sim, C.J.; Shin, J. Bis (indole) alkaloids as sortase A inhibitors from the sponge Spongosorites sp. Bioorg. Med. Chem. Lett. 2005, 15, 4927-4931. [CrossRef] [PubMed]

12. Burres, N.S.; Barber, D.A.; Gunasekera, S.P.; Shen, L.L.; Clement, J. Antitumor activity and biochemical effects of topsentin. J. Biochem. Pharmacol. 1991, 42, 745-751. [CrossRef]

13. Casapullo, A.; Bifulco, G.; Bruno, I.; Riccio, R. New Bisindole Alkaloids of the Topsentin and Hamacanthin Classes from the Mediterranean Marine Sponge Rhaphisia lacazei. J. Nat. Prod. 2000, 63, 447-451. [CrossRef] [PubMed]

14. Tsujii, S.; Rinehart, K.L.; Gunasekera, S.P.; Kashman, Y.; Cross, S.S.; Lui, M.S.; Pomponi, S.A.; Diaz, M.C. Topsentin, bromotopsentin, and dihydrodeoxybromotopsentin: Antiviral and antitumor bis(indolyl)imidazoles from Caribbean deep-sea sponges of the family Halichondriidae. Structural and synthetic studies. J. Org. Chem. 1988, 53, 5446-5453. [CrossRef]

15. Bokesch, H.R.; Pannell, L.K.; McKee, T.C.; Boyd, M.R. Coscinamides A, B and C, three new bis indole alkaloids from the marine sponge Coscinoderma sp. Tet. Lett. 2000, 41, 6305-6308. [CrossRef]

16. Xiaofei, J.; Wang, Z.; Dong, J.; Liu, Y.; Lu, A.; Wang, Q. Discovery of topsentin alkaloids and their derivatives as novel antiviral and anti-phytopathogenic fungus agents. J. Agric. Food Chem. 2016, 64, 9143-9151.

17. Willoughby, P.H.; Jansma, M.J.; Hoye, T.R. A guide to small-molecule structure assignment through computation of $\left({ }^{1} \mathrm{H}\right.$ and $\left.{ }^{13} \mathrm{C}\right)$ NMR chemical shifts. Nat. Protoc. 2014, 9, 643-660. [CrossRef] [PubMed]

18. Frisch, M.J.; Trucks, G.W.; Schlegel, H.B.; Scuseria, G.E.; Robb, M.A.; Cheeseman, J.R.; Scalmani, G.; Barone, V.; Petersson, G.A.; Nakatsuji, H.; et al. Gaussian 16 Rev. B.01, Wallingford, CT, 2016. Available online: https://gaussian.com/g16new/ (accessed on 29 October 2019).

19. Tomasi, J.; Mennucci, B.; Cammi, R. Quantum mechanical continuum solvation models. Chem. Rev. 2005, 105, 2999-3094. [CrossRef] [PubMed]

20. Bruhn, T.; Schaumloeffel, A.; Hemberger, Y.; Bringmann, G. SpecDis: Quantifying the comparison of calculated and experimental electronic circular dichroism spectra. Chirality 2013, 25, 243-249. [CrossRef] [PubMed]

Sample Availability: Samples of the compounds 1-8 are stored in the chemical repository at the Marine Institute (Co. Galway) and are available from the authors.

(C) 2019 by the authors. Licensee MDPI, Basel, Switzerland. This article is an open access article distributed under the terms and conditions of the Creative Commons Attribution (CC BY) license (http://creativecommons.org/licenses/by/4.0/). 